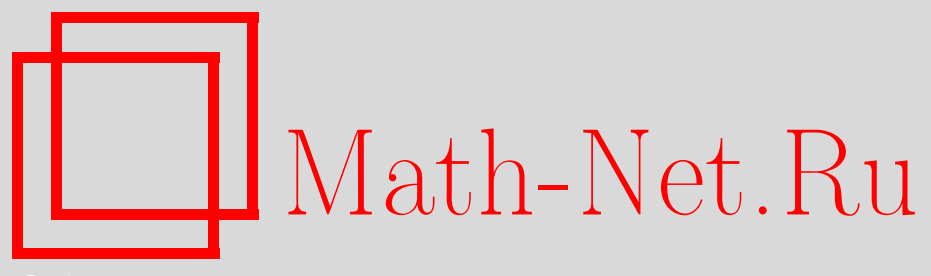

И. В. Павлов, О. В. Назарько, О неотрицательных адаптированных последовательностях случайных величин, являющихся процессами плотностей для деформированных стохастических базисов 1-го рода, УМН, 2015, том 70, выпуск 1, 185-186

DOI: https://doi.org/10.4213/rm9643

Использование Общероссийского математического портала Math-Net.Ru подразумевает, что вы прочитали и согласны с пользовательским соглашением http: //www . mathnet.ru/rus/agreement

Параметры загрузки:

IP: 54.81 .137 .203

26 апреля 2023 г., 15:58:27

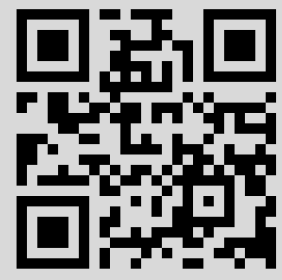




\title{
О неотрицательных адаптированных последовательностях случайных величин, являющихся процессами плотностей для деформированных стохастических базисов 1-го рода
}

\author{
И. В. Павлов, О. В. Назарько
}

Пусть $(\Omega, \mathbf{F})$ - фильтрованное пространство с дискретным временем, где $\Omega$ - произвольное множество, а $\mathbf{F}=\left(\mathscr{F}_{n}\right)_{n=0}^{\infty}-$ фильтрация. Семейство $\mathbf{Q}=\left(Q^{(n)}, \mathscr{F}_{n}\right)_{n=0}^{\infty}$ вероятностных мер $Q^{(n)}$, определенных на $\mathscr{F}_{n}$, называется деформацией 1-го рода (D1), если при всех $n \in \mathbb{N}=\{0,1,2, \ldots\}$ выполняются соотношения абсолютной непрерывности $\left.Q^{(n+1)}\right|_{\mathscr{F}_{n}} \ll Q^{(n)}$, и слабой деформацией (WD), если выполняются соотношения $\left.Q^{(n+1)}\right|_{\mathscr{F}_{n}} \sim Q^{(n)}$. Тройка $(\Omega, \mathbf{F}, \mathbf{Q})$ называется деформированным стохастическим базисом (соответственно 1-го рода и слабо деформированным). Ряд результатов, которые использованы в данной работе, можно найти в [1].

Для D1 $\mathbf{Q}$ введем процесс плотностей $\left(h_{n}, \mathscr{F}_{n}\right)_{n=0}^{\infty}$ согласно формуле $\left.d Q^{(n+1)}\right|_{\mathscr{F}_{n}}=$ $h_{n} d Q^{(n)}$. В настоящей работе изучаются следующие вопросы. Основной вопрос: при каких условиях на адаптированный неотрицательный определенный всюду на $\Omega$ процесс $\left(h_{n}, \mathscr{F}_{n}\right)_{n=0}^{\infty}$ существует D1 $\mathbf{Q}$, удовлетворяющая приведенному выше равенству, и как описать такие D1? На этот вопрос дается ответ в случае, когда $\sigma$-алгебра $\mathscr{F}_{n}$ для любого $n \in \mathbb{N}$ порождена разбиением $\Omega$ на не более чем счетное число атомов. Вспомогательный вопрос: как естественным образом деформации 1-го рода сводить к слабым деформациям? На этот вопрос дан ответ без существенных ограничений на фильтрацию. Отметим, что результаты данной работы важны для развития теории арбитража на финансовых $(B, S)$-рынках, заданных на деформированных стохастических базисах 1-го рода.

Начнем с вспомогательного вопроса. Пусть $\tau: \Omega \rightarrow \mathbb{N}$ - марковский момент (м.м.) относительно F. Для $A \in \mathscr{F}_{\tau}$ обозначим $Q^{(\tau)}(A)=\sum_{i=0}^{\infty} Q^{(i)}(A\{\tau=i\})$. Ясно, что $Q^{(\tau)}$ - неотрицательная $\sigma$-конечная мера, совпадающая на $\{\tau=n\}$ при любом $n \in \mathbb{N}$ с мерой $Q^{(n)}$. Если м. м. $\tau$ ограничен, то мера $Q^{(\tau)}$ ограничена и не является нулевой. Далее нам понадобится D1 R $=\left(R^{(n)}:=Q^{(\tau \wedge n)} / Q^{(\tau \wedge n)}(\Omega), \mathscr{F}_{\tau \wedge n}\right)_{n=0}^{\infty}$.

Процессы $\mathbf{Z}=\left(Z_{n}, \mathscr{F}_{n}\right)_{n=0}^{\infty}$ и $\mathbf{Z}^{\prime}=\left(Z_{n}^{\prime}, \mathscr{F}_{n}\right)_{n=0}^{\infty}\left(\right.$ соответственно м. м. $\tau$ и $\left.\tau^{\prime}\right)$ называются Q-неразличимыми, если $Z_{n}=Z_{n}^{\prime} Q^{(n)}$-п. н. (соответственно $Q^{(n)}(\{\tau=n\} \Delta$ $\left.\left\{\tau^{\prime}=n\right\}\right)=0$ ) для любого $n \in \mathbb{N}$. Если $\sigma$-алгебра $\mathscr{F}_{n}$ для любого $n \in \mathbb{N}$ полна относительно $Q^{(n)}$, то в случае неразличимости $\tau, \tau^{\prime}$ и $\mathbf{Z}, \mathbf{Z}^{\prime}$ имеем: $\mathscr{F}_{\tau \wedge n}=\mathscr{F}_{\tau^{\prime} \wedge n}$, $Q^{(\tau \wedge n)}=Q^{\left(\tau^{\prime} \wedge n\right)}$ и $Z_{\tau \wedge n}=Z_{\tau^{\prime} \wedge n}^{\prime} Q^{(\tau \wedge n)}$-п. в.

Пусть $\mathbf{Q}-\mathrm{D} 1$, а $\left(h_{n}, \mathscr{F}_{n}\right)_{n=0}^{\infty}-$ какая-либо фиксированная версия ее процесса плотностей. Рассмотрим м.м. $\tau=\inf \left\{n \geqslant 0: h_{n}=0\right\}$. Можно показать, что $Q^{(n)}(\tau \geqslant n)=1 \forall n \in \mathbb{N}$. С применением этого свойства доказывается следующая теорема.

Теорема 1. Пусть б-алгебра $\mathscr{F}_{n}$ для любого $n \in \mathbb{N}$ полна относителъно $Q^{(n)}$. Тогда деформачия $\mathbf{R}$ не зависит от версии прочесса плотностей деформачии 1-го рода $\mathbf{Q}$ и является слабой деформачией. При этом для любого деформированного субмартингала 1-го рода (см. [1]) $\left(Z_{n}, \mathscr{F}_{n}, Q^{(n)}\right)_{n=0}^{\infty}$ процесс $\left(Z_{\tau \wedge n}, \mathscr{F}_{\tau \wedge n}, R^{(n)}\right)_{n=0}^{\infty}$ есть слабо деформированный субмартингал, также не зависящий от версии $\left(h_{n}, \mathscr{F}_{n}\right)_{n=0}^{\infty}$.

Перейдем теперь к основному вопросу данной работы. Пусть $\sigma$-алгебра $\mathscr{F}_{n}$ для любого $n \in \mathbb{N}$ порождена разбиением $\Omega$ на не более чем счетное число атомов, $\mathscr{F}_{0}=$ $\{\Omega, \varnothing\}$. Через $\mathscr{D}_{n}$ обозначим множество всех атомов $\sigma$-алгебры $\mathscr{F}_{n}$. Мы будем строить деформации $\mathbf{Q}$ 1-го рода, порожденные адаптированным неотрицательным процессом $\left(h_{n}, \mathscr{F}_{n}\right)_{n=0}^{\infty}$. Значение случайной величины $h_{n}$ на атоме $D \in \mathscr{D}_{n}$ обозначим

Работа выполнена при поддержке РФФИ (гранты № 13-01-00637-а, 13-07-13159).

DOI: $10.4213 / \mathrm{rm} 9643$ 
через $h_{n}(D)$. При этом необходимо выполнение условия $h_{0}(\Omega)=1$. Рассмотрим м. м. $\tau=\inf \left\{n \geqslant 0: h_{n}=0\right\}$. Не нарушая общности, предположим, что $h_{n}=0$ при $n \geqslant \tau$ и $h_{n} \not \equiv 0$ для любого $n \in \mathbb{N}$.

Пусть $D_{n+1} \in \mathscr{D}_{n+1}$. Через $D_{k}, k=0,1, \ldots, n$, обозначим атом из $\mathscr{D}_{k}$ такой, что $D_{k} \supset D_{n+1}$. Ясно, что $\Omega=D_{0} \supset D_{1} \supset D_{2} \supset \cdots \supset D_{n} \supset D_{n+1}$. Обозначим через $\left(D_{n+1}^{i}\right)_{1 \leqslant i<m+1}$ совокупность всех атомов из $\mathscr{D}_{n+1}$ таких, что $\sum_{i=1}^{m} D_{n+1}^{i}=D_{n}$. Отметим, что $m=m\left(D_{n}\right)$ может равняться $\infty$ и что среди атомов $\left(D_{n+1}^{i}\right)_{1 \leqslant i<m+1}$ присутствует $D_{n+1}$.

Деформацию $\mathbf{Q} 1$-го рода с процессом плотностей $\left(h_{n}, \mathscr{F}_{n}\right)_{n=0}^{\infty}$ назовем невырожденной, если для любого $n \in \mathbb{N}$ и любого $D_{n} \in \mathscr{D}_{n}$ такого, что $h_{n}\left(D_{n}\right)>0$, выполняются неравенства $Q^{(n+1)}\left(D_{n+1}^{i}\right)>0,1 \leqslant i<m+1$. Множество всех невырожденных деформаций, порожденных процессом $\left(h_{n}, \mathscr{F}_{n}\right)_{n=0}^{\infty}$, обозначим $\mathbf{D}\left(h_{n}\right)$.

Пусть $\left(p_{n}, \mathscr{F}_{n}\right)_{n=0}^{\infty}-$ произвольный строго положительный процесс. Определим семейство мер $\mathbf{Q}=\left(Q^{(n)}, \mathscr{F}_{n}\right)_{n=0}^{\infty}$ (вообще говоря, $\sigma$-конечных) следующими формулами: $Q^{(0)}(\Omega)=1$ и $Q^{(n+1)}\left(D_{n+1}\right):=p_{n+1}\left(D_{n+1}\right) h_{n}\left(D_{n}\right) Q^{(n)}\left(D_{n}\right) \forall n \in \mathbb{N}, \forall D_{n} \in \mathscr{D}_{n}$.

Теорема 2. Для того чтобы неотрицателъный процесс $\left(h_{n}, \mathscr{F}_{n}\right)_{n=0}^{\infty}$ являлся процессом плотностей некоторой невырожденной $\mathrm{D} 1 \mathbf{Q}$, необходимо и достаточно (соответственно достаточно), чтобы для любого $n \in \mathbb{N}$ случайный прочесс $\left(p_{n}, \mathscr{F}_{n}\right)_{n=0}^{\infty}$, $p_{0}=1$, удовлетворял условиям: $\sum_{\forall D_{n+1} \in \mathscr{D}_{n+1}} \prod_{k=0}^{n} p_{k+1}\left(D_{k+1}\right) h_{k}\left(D_{k}\right)=1$ (где суммирование производится по всем атомам $\left.D_{n+1} \in \mathscr{D}_{n+1}\right) u \sum_{i=1}^{m} p_{n+1}\left(D_{n+1}^{i}\right)=1$ для всех $D_{n} \in \mathscr{D}_{n}$ таких, что $h_{n}\left(D_{n}\right)>0$ (соответственно удовлетворял последнему условию и равенствам $\sum_{i=1}^{m} h_{n+1}\left(D_{n+1}^{i}\right) p_{n+1}\left(D_{n+1}^{i}\right)=1$ для всех $D_{n} \in \mathscr{D}_{n}$ таких, что $h_{n}\left(D_{n}\right)>0$, - эти равенства выделяют класс канонических D1).

Теорема 3. Пусть $\mathbf{Z}=\left(Z_{n}, \mathscr{F}_{n}\right)_{n=0}^{\infty}-$ произвольный случайный процесс. Для невырожденной деформачии 1-го рода $\mathbf{Q} \in \mathbf{D}\left(h_{n}\right)$ следующие условия равносилъны:

1) $\mathbf{Q}$ - мартингальная деформачия 1-го рода $\left(\right.$ m.е. $\left(Z_{n}, \mathscr{F}_{n}, Q^{(n)}\right)_{n=0}^{\infty}-$ деформированный мартингал 1-го рода);

2) $\sum_{\forall D_{n} \in \mathscr{D}_{n}}\left|Z_{n}\left(D_{n}\right)\right| \prod_{k=0}^{n-1} p_{k+1}\left(D_{k+1}\right) h_{k}\left(D_{k}\right)<\infty$ для любого $n \geqslant 1$, а также $\sum_{i=1}^{m} Z_{n+1}\left(D_{n+1}^{i}\right) p_{n+1}\left(D_{n+1}^{i}\right)=Z_{n}\left(D_{n}\right)$ для любого $n \in \mathbb{N}$ и любого $D_{n} \in \mathscr{D}_{n}$ такого umo $h_{n}\left(D_{n}\right)>0$.

СледСтвиЕ 1. Если $\mathbf{Z}$ ограничен, то все канонические мартингальные деформации можно найти, решив для всех $n \in \mathbb{N}$ и всех $D_{n} \in \mathscr{D}_{n}$ таких, что $h_{n}\left(D_{n}\right)>0$, следующие системы:

$$
\left\{\begin{array}{l}
\sum_{i=1}^{m} x_{i}=1, \quad \sum_{i=1}^{m} h_{n+1}\left(D_{n+1}^{i}\right) x_{i}=1, \\
\sum_{i=1}^{m} Z_{n+1}\left(D_{n+1}^{i}\right) x_{i}=Z_{n}\left(D_{n}\right), \quad x_{i}>0 \forall i<m+1 .
\end{array}\right.
$$

\section{Список литературы}

[1] И. В. Павлов, О. В. Назарько, УМН, 68:6(414) (2013), 175-176.

\section{Игорь Викторович Павлов}

(Igor V. Pavlov)

Ростовский государственный строительный университет

E-mail: pavloviv2005@mail.ru

\section{Ольга Валерьевна Назарько}

(Olga V. Nazarko)

Ростовский государственный строительный университет

E-mail: ca_va@inbox.ru
Представлено Д. В. Трещёвым Принято редколлегией 19.08.2014 